\title{
Organización del mantenimiento preventivo en las gerencias de bienes y servicios de CORPOELEC región- occidental
}

\author{
Organization of preventive maintenance in the goods and services \\ management of CORPOELEC region- western
}

\author{
Manuel Cedeño \\ manuelcedeño89@gmail.com \\ Código ORCID: 0000-0002-0125-8551 \\ Universidad del Zulia, Venezuela
}

\begin{abstract}
Resumen
El estudio buscó describir la organización del mantenimiento preventivo aplicado en las gerencias de bienes y servicios de CORPOELEC Región Occidental. Metodológicamente se tipificó como descriptiva, con diseño no experimental, transeccional y de campo. La población estuvo constituida por los departamentos de flota de las gerencias de bienes y servicios de CORPOELEC Región Occidental. La técnica de recolección de datos fue la encuesta y el instrumento un cuestionario con escala de frecuencia, compuesto por 12 ítems, se utilizó el juicio de expertos para la validez y el coeficiente Alfa Cronbach para su confiabilidad, cuyo resultado fue 0,867. La media aritmética y la desviación estándar se usaron para el análisis de los resultados. Se concluyó, que dentro del proceso, los indicadores descentralización, autoridad, y organigrama se aplican dentro del objetivo. Sin embargo se requiere mejorar el resultado obtenido en el promedio del indicador funciones puesto que éste tiene baja aplicación.
\end{abstract}

Palabras clave: autoridad, descentralización, funciones, mantenimiento preventivo, organigrama, organización del mantenimiento

\begin{abstract}
The study sought to describe the organization of preventive maintenance applied in the management of goods and services of CORPOELEC Western Region. Methodologically it was typified as descriptive, with a non-experimental, transectional and field design. The population was made up of the fleet departments of the departments of goods and services of CORPOELEC Western Region. The data collection technique was the survey and the instrument was a questionnaire with a frequency scale, made up of 12 items, using expert judgment for validity and the Alpha Cronbach coefficient for its reliability, the result of which was 0.867 . The arithmetic mean and standard deviation were used for the analysis of the results. It was concluded that within the process, the indicators decentralization, authority, and organizational chart are applied within the objective. However, it is necessary to improve the result obtained in the average of the functions indicator since it has low application.
\end{abstract}

Keywords: authority, decentralization, functions, preventive maintenance, organization chart. maintenance oraanization 


\section{INTRODUCCIÓN}

A nivel mundial las empresas deben afrontar retos en cuanto al mantenimiento de equipos, para garantizar su operatividad. Esto se logra, desarrollado e implementando técnicas con el objeto de optimizar los procesos de mantenimiento a fin de elevar los estándares de calidad, productividad, seguridad y utilidad financiera. En fin, estas técnicas deben responder a nuevos conceptos y expectativas, donde la cantidad y complejidad de los sistemas a mantener crece cada día.

Visto las empresas como sistemas complejos e independientemente de sus objetos, están constantemente inmersas en una dinámica en donde se encuentran involucrados proyectos, personas y tecnologías. Éstas están expuestas a mejorar cada vez más sus capacidades para producir los bienes y servicios que la sociedad actual demanda con altas exigencias de calidad y desempeño.

Con base a este desarrollo de la actividad productiva, se ha requerido siempre del apoyo de la gestión de mantenimiento para cualquier actividad que no tenga que contar con el personal de este departamento, bien sea para garantizar que la producción de la organización no sea afectada por fallas imprevistas o mejorar la productividad, confiabilidad y disponibilidad de los equipos para ser más competitivos en las actividades de mantenimiento.

En tal sentido, en la época actual, debido a las consideraciones demandadas por el mercado, se encuentra en un estado de transición en la que la excelencia es considerada parte del producto, por ello sería inconcebible que el mantenimiento, siendo función importante de apoyo a la producción, y por ende parte de la organización empresarial, no la tuviera ni eventualmente, así las cosas, las empresas tienen latente el reto de cómo mejorar sus actividades de gestión del mantenimiento para ser más sostenibles.

De igual manera, la gestión de mantenimiento, implica disponer de una metodología que involucre sistemas de información y una acción cíclica de mejoramiento constituida por auditorías de los puntos críticos de éxito en mantenimiento, planificación a la medida y ejecución del plan de trabajo definido aplicando las herramientas de gestión apropiadas.

En la actualidad, el mantenimiento en las empresas es considerado como la mejor alternativa económica en sus procesos productivos, transformándose en una actividad cada vez más importante dentro de los complejos industriales ya sea a nivel internacional, nacional y local. Proyectar al mantenimiento como un gasto significa no invertir en los activos de la empresa. Esta transformación que está ocurriendo en este mundo globalizado ha hecho evidente la necesidad de una mejora sustancial y sostenida de los resultados operacionales como los financieros de las empresas.

De acuerdo con Garrido (2003), el mantenimiento se define como un conjunto de técnicas destinado a conservar equipos $e$ instalaciones en servicio durante el mayor tiempo posible, buscando la más alta disponibilidad y con el máximo rendimiento. Por otra parte, el autor mencionado explica que, es una metodología de análisis sistemático, objetivo y documentado que puede ser aplicado a cualquier tipo de instalación industrial, útil para el desarrollo o mejoramiento de un plan de mantenimiento.

En este punto, la situación actual del país exige a las empresas Venezolanas la adaptación de un equipo multidisciplinario de trabajo que se encargue de optimizar la confiabilidad operacional de un sistema que funciona bajo las condiciones de trabajo definidas, estableciendo las actividades más efectivas de mantenimiento en función de la criticidad de los activos pertenecientes a dicho sistema tomando en cuenta los posibles efectos que originan los modos de fallas de estos activos a la seguridad, al ambiente y a las operaciones. 
En este marco referencial, se contextualizan las empresas de servicios que operan en Venezuela, específicamente en el Estado Zulia. Entre ellas, CORPOLEC que es una empresa operadora estatal adscrita al Ministerio del Poder Popular para la Energía y Petróleo, encargada de la realización de las actividades de generación, transmisión, distribución y comercialización de potencia y energía eléctrica.

Según Decreto-Ley $N^{\circ} 5.330$ (2007), la empresa CORPOELEC se encuentra conformada por las siguientes empresas de generación, transmisión, distribución y comercialización de energía eléctrica: Electrificación del Caroní, C.A. (EDELCA), Energía Eléctrica de Venezuela, S.A. (ENELVEN), Empresa Nacional de Generación C.A: (ENAGER), Compañía de Administración y Fomento Eléctrico S.A. (CADAFE), Energía Eléctrica de la Costa Oriental del Lago C.A: (ENELCO), Energía Eléctrica de Barquisimeto S.A. (ENELBAR), Sistema Eléctrico del Estado Nueva Esparta (SENECA) y la Electricidad de Caracas (La EDC).

Estas empresas, a juicio del investigador, se han visto afectadas por el entorno económico e industrial actual del estado venezolano, al afirmar que la profundidad y amplitud del cambio en el ámbito productivo, tanto en maquinaria como en instalaciones, métodos de organización y trabajo, involucrando las actividades de mantenimiento, se han visto afectadas por algunas fallas que se presentan por la falta de repuestos, personal no capacitado, distribución de un área de trabajo inadecuada, así como la falta de información técnica de los equipos en cuanto a manuales de operación y de mantenimiento.

Enmarcado en este contexto, en el departamento de flota de la gerencia de bienes y servicios de CORPOELEC Región occidental, en ejecución de sus labores, según la observación directa por parte del investigador, se evidencia la falta de un plan de mantenimiento según carga de trabajo y horas de uso de los vehículos. Así como un mantenimiento de primer nivel que lo hace el propio usuario, por lo que siempre se hace a tiempo, en este caso, no es necesario llamar a nadie ni interfiere en la producción, sólo se requiere de formación y delimitación de las funciones del usuario, resultando así lo que se conoce como el mantenimiento preventivo.

Según Duffuaa y otros (2007), el mantenimiento preventivo, refiere a una serie de tareas planeadas previamente que se llevan a cabo para contrarrestar las causas conocidas de fallas potenciales de las funciones para las que fue creado un activo. Puede planearse $y$ programarse con base en el tiempo, el uso o la condición del equipo.

Ahora bien, según la Norma Covenín 3049-93 (1993), la gestión de mantenimiento preventivo es el que utiliza todos los medios disponibles, incluso los estadísticos, para determinar la frecuencia de las inspecciones, revisiones, sustitución de piezas claves, probabilidad de aparición de averías, vida útil, $u$ otras. Su objetivo es adelantarse a la aparición o predecir la presencia de las fallas.

Desde este contexto y de acuerdo con lo que se pudo conocer mediante conversaciones con empleados de las gerencias de bienes y servicios además de los registros llevados por los departamentos de transporte de la empresa COPOLEC ubicada en los Estados Zulia, Trujillo y Falcón, se detectó que se encuentran debilidades en la gestión del mantenimiento preventivo, debido a que no se tienen definidas las normas, lineamientos organizacionales, políticas, y objetivos estratégicos. Asimismo, se evidencia falta de compromiso del personal en sus diversos niveles jerárquicos, ausencia de gestión, seguimiento y control del mantenimiento preventivo, falta de manuales de normas y procedimientos del mantenimiento preventivo, entre otras.

Por otro lado, los departamentos en estudio no tienen control en los procesos, al igual que al momento de efectuar un mantenimiento 
preventivo no cuentan con la suficiente confiabilidad crediticia con los proveedores por los incumplimientos constantes de CORPOELEC al momento del pago de los servicios contratados y si se efectúa el pago se hace en tiempos que no es rentable para la prestadora del servicio contratado, a pesar de que se logró una alianza institucional con la industria Nacional Petróleo de Venezuela (PDVSA) para la dotación de lubricantes, ésta no se hace de forma oportuna y continua, aunado a ésto no se cuenta con los filtros necesarios para realizar los mantenimiento con mano de obra propia teniendo que recurrir en muchas ocasiones, a los mismos usuarios para que costeen estos gastos.

De continuar esta situación, la empresa podría presentar un desmejoramiento en la disponibilidad del servicio a la comunidad a los clientes, elevación de costos tanto operativos como de mantenimiento, constituyéndose en un grave problema para las mismas. Desde esa perspectiva se realiza, a través de esta investigación una descripción de la organización del mantenimiento preventivo aplicado en las gerencias de bienes y servicios de CORPOELEC Región Occidental.

\section{Mantenimiento preventivo}

Nava (2008: p. 53), establece que el mantenimiento preventivo es "la actividad planeada programada para ajustar, reparar o cambiar partes en equipo ante de que ocurra las falla o daño mayores", reduciendo así los costos generado en mantenimiento. En este concepto está implícita la necesidad de parada de equipo o sistema para realizar un trabajo programado.

Asimismo, Duffuaa y otros (2007) expresan que básicamente la gestión de mantenimiento preventivo consiste en programar revisiones de los equipos, apoyándose en el conocimiento de la máquina en base a la experiencia y los históricos obtenidos de las mismas. Se confecciona un plan de mantenimiento para cada máquina, donde se realizarán las acciones necesarias: engrasar, cambiar correas, desmontaje, limpieza, entre otros.

En el mismo orden de ideas, para Creus (2006), el mantenimiento preventivo consiste en inspeccionar o revisar los equipos, instalaciones o estructuras, para evitar que se produzca un paro por a vería o de que exista una anomalía que afecte de forma significativa a la cantidad o calidad del producto acabado, $\circ$ a las condiciones de seguridad derivadas de la utilización del equipo.

Así las cosas, la aplicación del mantenimiento preventivo supone asegurar el correcto funcionamiento de los equipos e instalaciones, y por lo tanto su rendimiento y sus prestaciones durante toda su vida útil y consecuentemente reduciendo los posibles accidentes provocados por el mal estado de los mismos.

En este contexto de ideas, González (2011), indica que la gestión de mantenimiento preventivo consiste en realizar ciertas reparaciones, cambios de componentes, piezas, según intervalos de tiempo, por determinados criterios, prefijados para reducir la probabilidad de avería, determinados criterios, o pérdida de rendimiento de un ítem.

Visto así, para el investigador la gestión de mantenimiento preventivo consiste en la inspección periódica previamente planificada con el propósito de reparar o sustituir, piezas de una maquinaria o equipo, incluso aunque no muestre signos de mal funcionamiento, y de esta manera evitar posibles irregularidades que afectan la comunidad operativa de una organización.

\section{Organización del mantenimiento}

Duffuaa, y otros (2007), señalan que la organización de un sistema de mantenimiento es aquella que incluyen el diseño de trabajo, los estándares de tiempo y la administración de proyectos como actividades de la organización de mantenimiento. El diseño del trabajo abarca el contenido de cada tarea y 
determina el método que se va a utilizar, las herramientas especiales necesarias y los trabajadores calificados requeridos para el cumplimiento de sus funciones.

En el mismo orden de ideas para Milano (2010), la organización del mantenimiento puede ser a través de estructuras funcionales (centralizadas) posicional (descentralizada). Las estructuras funcionales comprenden la organización simple y la organización funcional. La organización funcional agrupa a las personas en base a la experiencia comunes, en este tipo de organización cada rectángulo del organigrama representa una especialización funcional diferente. En el caso del mantenimiento la organización se puede distribuir en planificación y control, ingeniería de contabilidad, estudio de fallas, control de gestión, entre otros.

Así las cosas, no existe organización de mantenimiento básica que pueda ser utilizada en todos los casos. La organización en general es una combinación voluntaria de personas y recursos diseñados para satisfacer situaciones específicas técnicas, geográficas y de personal, dirigida a la conservación de objetivos y fines, caracterizada por un sistema de interrelaciones que se producen entre los elementos que la componen.

De acuerdo a los autores ante mencionados, el investigador señala la organización de mantenimientos como aquella que agrupa todas las funciones y actividades en unidades específicas, con base en su similitud. Además, debe existir una coordinación, sincronizando los recursos y los esfuerzos de un grupo social, con el fin de lograr oportunidad, unidad, armonía y rapidez, en desarrollo de los objetivos de la organización.

\section{Descentralización}

Duffuaa y otros (2007), la define como aquella que ocurre un cambio organizacional donde la toma de decisiones y las responsabilidades de tareas son transferidas y asignadas a los niveles inferiores dentro de la jerarquía de una organización, para que la ejecución de las actividades sea más apropiada a las características, particulares dentro de una empresa. La descentralización de la función de mantenimiento puede producir un tiempo de respuestas más rápido y lograr que los trabajadores se familiaricen más con los problemas de la empresa.

De igual manera, Koontz y Weihrich (2013), definen la descentralización como la tendencia a distribuir la autoridad de toma de decisiones en una estructura organizada. Este es un aspecto fundamental de la delegación, en la medida en que no se delega autoridad, se le centraliza. La descentralización implica algo más que delegación, es reflejo de la filosofía de una organización y de su dirección.

Para los autores Robbins y Decenzo (2002), esta acción refleja una especial filosofía de administración y obliga a saber con detalles, que decisiones y actividades se llevaran a cabo en otros niveles de la organización y cuales deberán permanecer en los niveles superiores, así como diseñar políticas, para orientar la toma de decisiones, seleccionar y capacitar a los colaboradores y establecer los registros y los controles adecuados.

A fines de la investigación, el investigador refiere que la descentralización es una tendencia organizativa de la administración pública, conforme a la cual se confiere personalidad jurídica propia a ciertos entes a los que se les otorga autonomía orgánica relativa, respecto del órgano central.

\section{Organigrama}

Robbins y Decenzo (2002), refieren que el organigrama es uno de los resultados de la organización, pues es la representación gráfica de cómo se organizó la empresa, es un conjunto de figuras geométricas que representan órganos y líneas que se utilizan para dar una idea gráfica de cómo está estructurada una organización, por ello es de gran utilidad en la empresa.\} 
Para los autores Duffuaa y otros (2007) el organigrama es de gran importancia ya que a través de éste se puede visualizar la dependencia de la organización dentro de la empresa, es así como la función de mantenimiento puede depender de la gerencia general $\circ$ del departamento de producción directamente.

También la norma COVENÍN 3049-93 establece el organigrama o estructura de mantenimiento como la descomposición, localización y arreglo de los recursos para hacer frente de la mejor manera a una carga de trabajo esperada. En lo que se refiere a los organigramas los responsables de reorganizar cuando así lo demande las condiciones de la empresa son los líderes de alto nivel.

A efectos de la investigación, el organigrama a juicio del investigador, establece la división de los departamentos, las áreas y los cargos funcionales que se deben desempeñar dentro de una organización, ajustándolo de acuerdo a la necesidad que estas presenten y así poder lograr planificar las actividades que en cada una de ellas se deben ejecutar y cumplir.

\section{Autoridad}

Para Dufuua y otros (2007), se entiende por autoridad aquella persona que posee la propiedad de mandar y a la obligación de que sus decisiones tomadas sean cumplidas por otros dentro de un departamento o unidad en una organización, es decir, potestad que tiene una persona para tomar una decisión que produzcan efectos en su sistema.

Según Robbins y Decenzo, (2002), es el derecho inherente a una posición administrativa para dar órdenes y para esperar que sean obedecidas. La autoridad es el grado de libertad de acción en los puestos organizacionales que le confiere a las personas que los ocupan el derecho de usar su criterio al tomar decisiones

De igual manera, Koontz y Weihrich, (2013), consideran que es la facultad de mandar y la obligación correlativa de ser obedecido por otros dentro de un departamento o unidad. Una definición más completa sería la facultad para tomar decisiones que produzcan efectos en un sistema.

A juicio del investigador, la autoridad en una organización es el derecho propio de un puesto (y por lo tanto de la persona que lo ocupa) a ejercer discrecionalidad en la toma de decisiones que afectan a otras personas. Se trata, por supuesto, de un tipo de poder, pero en el marco de una organización.

\section{Funciones}

Robbins y Decenzo (2002), la definen como las actividades y tareas que deben realizarse para cumplir con un fin específico. Las funciones son tareas de administración, planeación, organización, integración del personal que se desarrollan para cumplir con objetivos prefijados.

Por su parte los autores Koontz y Weihrich, (2013) consideran que son las actividades y tareas en forma rutinaria y que al realizarlas, permitirán el cumplimiento de las acciones tácticas que acercan al logro de los objetivos estratégicos. El nivel de coordinación de estas funciones opera entre los niveles operativos y sirve de intermediación y coordinación entre ambos.

Visto así, el investigador considera que para que las organizaciones funcionen adecuadamente, necesitan ser organizadas, por esta razón es necesario señalar la importancia de ésta función, esta relaciona entre si las actividades necesarias y dispone quien debe desempeñarlas, establece la mejor forma de alcanzar los objetivos propuestos recordando que los objetivos planteados van relacionados según la función que desempeñe el grupo o departamento, para el alcance de estos objetivos combina las tareas en forma lógica y eficiente. 


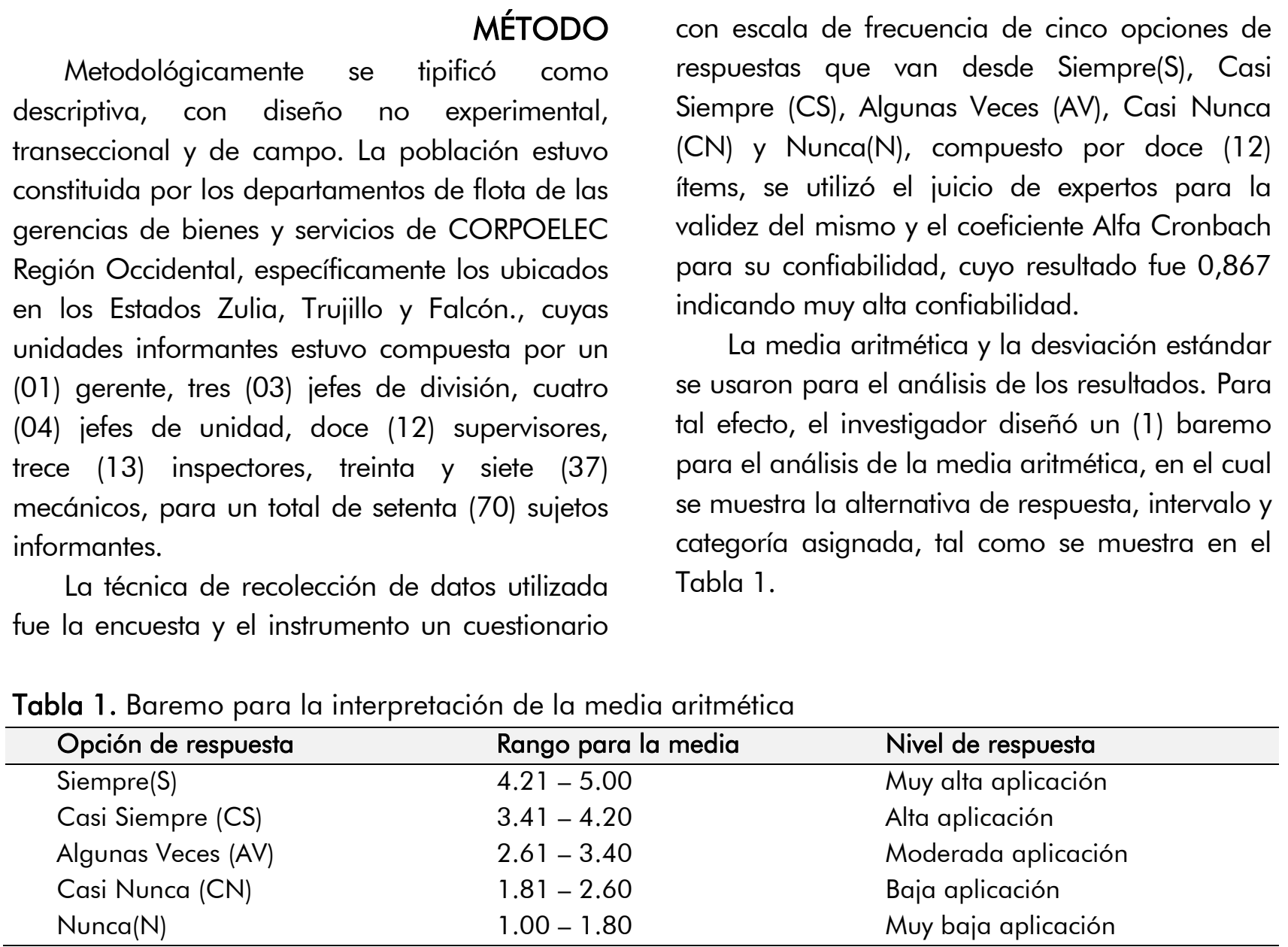

Fuente: Elaboración propia (2019)

Con respecto a la desviación estándar, la cual indica el grado de dispersión de las respuestas y su nivel de confiabilidad, en función de la escala de medición utilizada, tal como se muestra en el Tabla 2.

Tabla 2. Categoría de análisis para la interpretación de la desviación estándar

\begin{tabular}{cl}
\hline Rango & \multicolumn{1}{c}{ Descripción } \\
\hline $2.01-2.50$ & Muy alta dispersión de las respuestas y, muy baja confiabilidad de las mismas. \\
$1.51-2.00$ & Alta dispersión de las respuestas y baja confiabilidad de las mismas. \\
$1.01-1.50$ & Moderada dispersión de las respuestas y, moderada confiabilidad de las mismas. \\
$0.51-1.00$ & Baja dispersión de las respuestas y alta confiabilidad de las mismas. \\
$0.00-0.50$ & Muy baja dispersión de las respuestas y, muy alta confiabilidad de las mismas \\
\hline
\end{tabular}

Fuente: Elaboración propia (2019)

\section{RESULTADOS}

Como se evidencia en la tabla 3 , el indicador descentralización, muestra la media de 4,61 y desviación estándar de 0,48 ubicándose en la categoría de muy alta aplicación con muy baja dispersión de las respuestas y, muy alta confiabilidad de las mismas. Según estos resultados en el departamento de flota de las gerencias de bienes y servicios de CORPOELEC Región Occidental se conoce que decisiones y actividades se llevarán a cabo en otros niveles de la organización y cuales deberán permanecer en los niveles superiores. 
Tabla 3. Indicador Descentralización

\begin{tabular}{|c|c|c|c|c|}
\hline Ítems & $\overline{\bar{X}}$ & Categoría & $\sigma$ & Categoría \\
\hline $\begin{array}{l}\text { 1. Cuenta con autoridad para } \\
\text { planificar las actividades que } \\
\text { se llevarán a cabo en la } \\
\text { ejecución del mantenimiento } \\
\text { preventivo }\end{array}$ & 4,54 & $\begin{array}{l}\text { Muy alta } \\
\text { aplicación }\end{array}$ & 0,50 & $\begin{array}{l}\text { Muy baja dispersión de las } \\
\text { respuestas y, muy alta } \\
\text { confiabilidad de las mismas }\end{array}$ \\
\hline $\begin{array}{l}\text { 2. Toma las decisiones en las } \\
\text { actividades relacionadas a la } \\
\text { ejecución del mantenimiento } \\
\text { preventivo }\end{array}$ & 4,54 & $\begin{array}{l}\text { Muy alta } \\
\text { aplicación }\end{array}$ & 0,50 & $\begin{array}{l}\text { Muy baja dispersión de las } \\
\text { respuestas y, muy alta } \\
\text { confiabilidad de las mismas }\end{array}$ \\
\hline $\begin{array}{l}\text { 3. Garantiza la ejecución de las } \\
\text { actividades del mantenimiento } \\
\text { preventivo en el tiempo } \\
\text { planificado }\end{array}$ & 4,74 & $\begin{array}{l}\text { Muy alta } \\
\text { aplicación }\end{array}$ & 0,44 & $\begin{array}{l}\text { Muy baja dispersión de las } \\
\text { respuestas y, muy alta } \\
\text { confiabilidad de las mismas }\end{array}$ \\
\hline $\begin{array}{l}\text { Promedios de la media y desviación } \\
\text { estándar }\end{array}$ & 4,61 & $\begin{array}{l}\text { Muy alta } \\
\text { aplicación }\end{array}$ & 0,48 & $\begin{array}{l}\text { Muy baja dispersión de las } \\
\text { respuestas y, muy alta } \\
\text { confiabilidad de las mismas }\end{array}$ \\
\hline
\end{tabular}

Fuente: Elaboración Propia (2019)

De forma tal, se evidencia en estos resultados, muy alta aplicación al considerar los encuestados que cuentan con autoridad para planificar las actividades que se llevarán a cabo en la ejecución del mantenimiento preventivo $(4,54)$ con desviación $(0,50)$; toma las decisiones en las actividades relacionadas a la ejecución del mantenimiento preventivo $(4,54)$, con desviación $(0,50)$; garantiza la ejecución de las actividades del mantenimiento preventivo en el tiempo planificado $(4,74)$, con desviación $(0,44)$.

Los resultados se soportan de acuerdo al aporte de Duffuaa y otros (2007), quienes la definen como aquella que ocurre un cambio organizacional donde la toma de decisiones y las responsabilidades de tareas son transferidas $y$ asignadas a los niveles inferiores dentro de la jerarquía de una organización, para que la ejecución de las actividades sea más apropiada a las características, particulares dentro de una empresa. La descentralización de la función de mantenimiento puede producir un tiempo de respuestas más rápido y lograr que los trabajadores se familiaricen más con los problemas de la empresa.
De igual manera, se corresponde con lo expuesto por el investigador al referir que la descentralización es una tendencia organizativa de la administración pública, conforme a la cual se confiere personalidad jurídica propia a ciertos entes a los que se les otorga autonomía orgánica relativa, respecto del órgano central.

Con base a lo anterior, es importante considerar en la organización del mantenimiento que el supervisor sea el encargado de ejercer autoridad en la planificación y toma de decisiones en las actividades inherentes a la ejecución del mantenimiento preventivo, esto conllevará a que dichas actividades se ejecuten de la manera más apropiada y pueda producir tiempo de respuesta más rápido.

La tabla 4 muestra los resultados para el indicador organigrama, con una media de 3,02 y desviación de 0,46, indicando moderada aplicación así como muy baja dispersión de las respuestas y, muy alta confiabilidad de las mismas, refieren estos resultados que el departamento de flota de las gerencias de bienes y servicios de CORPOELEC Región Occidental, no siempre se tiene una idea de cómo está estructurada. 
Tabla 4. Indicador Organigrama

\begin{tabular}{|c|c|c|c|c|}
\hline Ítems & $\overline{\bar{X}}$ & Categoría & $\bar{\sigma}$ & Categoría \\
\hline $\begin{array}{l}\text { 4.- La gerencia tiene establecida una } \\
\text { estructura organizacional } \\
\text { claramente definida para el } \\
\text { departamento de flota }\end{array}$ & 2,26 & $\begin{array}{l}\text { Baja } \\
\text { aplicación }\end{array}$ & 0,44 & $\begin{array}{l}\text { Muy baja dispersión de las } \\
\text { respuestas y, muy alta } \\
\text { confiabilidad de las mismas }\end{array}$ \\
\hline $\begin{array}{l}\text { 5.- La gerencia tiene establecida la } \\
\text { descripción de cargos para el } \\
\text { personal que labora en el } \\
\text { departamento de flota }\end{array}$ & 2,26 & $\begin{array}{l}\text { Baja } \\
\text { aplicación }\end{array}$ & 0,44 & $\begin{array}{l}\text { Muy baja dispersión de las } \\
\text { respuestas y, muy alta } \\
\text { confiabilidad de las mismas }\end{array}$ \\
\hline $\begin{array}{l}\text { 6.- El personal que labora en el } \\
\text { departamento de flota tiene } \\
\text { conocimiento de las actividades } \\
\text { que debe desempeñar }\end{array}$ & 4,53 & $\begin{array}{l}\text { Muy alta } \\
\text { aplicación }\end{array}$ & 0,50 & $\begin{array}{l}\text { Muy baja dispersión de las } \\
\text { respuestas y, muy alta } \\
\text { confiabilidad de las mismas }\end{array}$ \\
\hline $\begin{array}{l}\text { Promedios de la media y desviación } \\
\text { estándar }\end{array}$ & 3,02 & $\begin{array}{l}\text { Moderada } \\
\text { aplicación }\end{array}$ & 0,46 & $\begin{array}{l}\text { Muy baja dispersión de las } \\
\text { respuestas y, muy alta } \\
\text { confiabilidad de las mismas }\end{array}$ \\
\hline
\end{tabular}

Fuente: Elaboración Propia (2019)

Sustentado en el nivel de moderada aplicación al referir los encuestados que la gerencia con baja aplicación tiene establecida una estructura organizacional claramente definida para el departamento de flota $(2,26)$, y la descripción de cargos para el personal que labora en el departamento de flota $(2,26)$, no obstante con muy alta aplicación el personal que labora en el departamento de flota tiene conocimiento de las actividades que debe desempeñar $(3,02)$. En todos los ítems se denota muy baja dispersión y muy alta confiabilidad de las respuestas emitidas $(0,44$; $0,44 ; 0,50)$.

El análisis de estos resultados no coincide totalmente con la teoría expuesta por la norma COVENÍN 3049-93, donde se establece el organigrama $\mathrm{O}$ estructura de mantenimiento como la descomposición, localización y arreglo de los recursos para hacer frente de la mejor manera a una carga de trabajo esperada. En lo que se refiere a los organigramas los responsables de reorganizar cuando así lo demande las condiciones de la empresa son los líderes de alto nivel

Tampoco tiene mayor coincidencia con lo expuesto por el investigador al referir éste que el organigrama establece la división de los departamentos, las áreas y los cargos funcionales que se deben desempeñar dentro de una organización, ajustándolo de acuerdo a la necesidad que estas presenten y así poder lograr planificar las actividades que en cada una de ellas se deben ejecutar y cumplir.

Por lo antes expuesto, dentro el departamento de flota de las gerencias de bienes y servicios de CORPOELEC Región Occidental, se debe considerar que el organigrama y la descripción de cargo son un papel fundamental para la organización del mantenimiento ya que facilitan la ejecución de los planes involucrados en las actividades del mantenimiento preventivo garantizando que se cumplan los mismos.

La tabla 5 muestra los resultados obtenidos al evaluar el indicador autoridad, con una media de 4,00 y desviación estándar de 0,42 ubicándose en la categoría de alta aplicación con muy baja dispersión de las respuestas y, muy alta confiabilidad de las mismas. Este resultado permite inferir que en el departamento de flota de las gerencias de bienes y servicios de CORPOELEC Región Occidental se tiene facultad de mandar y la obligación correlativa de ser obedecido por otros. 
Tabla 5. Indicador Autoridad

\begin{tabular}{|c|c|c|c|c|}
\hline Ítems & $\overline{\bar{X}}$ & Categoría & $\sigma$ & Categoría \\
\hline $\begin{array}{l}\text { 7.-Ejerce autoridad en la } \\
\text { ejecución de las actividades } \\
\text { del mantenimiento preventivo }\end{array}$ & 4,54 & $\begin{array}{l}\text { Muy alta } \\
\text { aplicación }\end{array}$ & 0,50 & $\begin{array}{l}\text { Muy baja dispersión de } \\
\text { las respuestas y, muy alta } \\
\text { confiabilidad de las } \\
\text { mismas }\end{array}$ \\
\hline $\begin{array}{l}\text { 8.-El personal involucrado en las } \\
\text { actividades del mantenimiento } \\
\text { preventivo tiene conocimiento } \\
\text { acerca de las decisiones } \\
\text { tomadas por la alta gerencia }\end{array}$ & 2,93 & $\begin{array}{l}\text { Moderada } \\
\text { aplicación }\end{array}$ & 0,26 & $\begin{array}{l}\text { Muy baja dispersión de } \\
\text { las respuestas y, muy alta } \\
\text { confiabilidad de las } \\
\text { mismas }\end{array}$ \\
\hline $\begin{array}{l}\text { 9.-Se garantiza que el personal } \\
\text { cumpla con las decisiones } \\
\text { tomadas a su cargo }\end{array}$ & 4,54 & $\begin{array}{l}\text { Muy alta } \\
\text { aplicación }\end{array}$ & 0,50 & $\begin{array}{l}\text { Muy baja dispersión de } \\
\text { las respuestas y, muy alta } \\
\text { confiabilidad de las } \\
\text { mismas }\end{array}$ \\
\hline $\begin{array}{l}\text { Promedios de la media y } \\
\text { desviación estándar }\end{array}$ & 4,00 & Alta aplicación & 0,42 & $\begin{array}{l}\text { Muy baja dispersión de } \\
\text { las respuestas y, muy alta } \\
\text { confiabilidad de las } \\
\text { mismas }\end{array}$ \\
\hline
\end{tabular}

Fuente: Elaboración Propia (2019)

El valor obtenido en esta media, es producto de una muy alta aplicación al considerar los encuestados que se ejerce autoridad en la ejecución de las actividades del mantenimiento preventivo $(4,54)$ y se garantiza que el personal cumpla con las decisiones tomadas a su cargo $(4,54)$; no así para el personal involucrado en las actividades del mantenimiento preventivo los cuales con moderada aplicación tienen conocimiento acerca de las decisiones tomadas por la alta gerencia $(2,93)$. En todos los ítems se denota muy baja dispersión y muy alta confiabilidad de las respuestas emitidas $(0,50 ; 0,26 ; 0,50)$.

Las afirmaciones anteriores, tienen su sustento en lo referido por Dufuua y otros (2007), para quienes se entiende por autoridad aquella persona que posee la propiedad de mandar y a la obligación de que sus decisiones tomadas sean cumplidas por otros dentro de un departamento o unidad en una organización, es decir, potestad que tiene una persona para tomar una decisión que produzcan efectos en su sistema.

Asimismo, concuerda los resultados en lo expuesto por el investigador, para quien la autoridad en una organización es el derecho propio de un puesto (y por lo tanto de la persona que lo ocupa) a ejercer discrecionalidad en la toma de decisiones que afectan a otras personas. Se trata, por supuesto, de un tipo de poder, pero en el marco de una organización.

Por lo antes mencionado, dentro del departamento de flota de las gerencias de bienes y servicios de CORPOELEC Región Occidental, la autoridad juega un papel fundamental para la organización del mantenimiento, ya que garantizará que la toma de decisiones en la ejecución de los planes involucrados en las actividades del mantenimiento preventivo se cumpla en pro del beneficio de la organización.

Con referencia al indicador funciones, la tabla 6 muestra los resultados, obteniéndose una media de 2,59 y una desviación estándar de 0,27 ubicándose en la categoría de baja aplicación, así como, muy baja dispersión de las respuestas y, muy alta confiabilidad de las mismas; lo que permite afirmar, el poco cumplimiento de las tareas necesarias para cumplir con objetivos prefijados. 
Tabla 6. Indicador Funciones

\begin{tabular}{|c|c|c|c|c|}
\hline Ítems & $\bar{X}$ & Categoría & $\sigma$ & Categoría \\
\hline $\begin{array}{l}\text { 10.- La gerencia cuenta con un } \\
\text { manual organizacional donde } \\
\text { se encuentren establecidas las } \\
\text { funciones que cada persona } \\
\text { debe desempeñar }\end{array}$ & 1,90 & $\begin{array}{l}\text { Baja } \\
\text { aplicación }\end{array}$ & 0,30 & $\begin{array}{l}\text { Muy baja dispersión de las } \\
\text { respuestas y, muy alta } \\
\text { confiabilidad de las mismas }\end{array}$ \\
\hline $\begin{array}{l}\text { 11.- El personal involucrado en las } \\
\text { actividades relacionadas a la } \\
\text { gestión del mantenimiento } \\
\text { preventivo tiene conocimiento } \\
\text { acerca de las funciones } \\
\text { establecidas en el manual } \\
\text { organizacional }\end{array}$ & 2,93 & $\begin{array}{l}\text { Moderada } \\
\text { aplicación }\end{array}$ & 0,26 & $\begin{array}{l}\text { Muy baja dispersión de las } \\
\text { respuestas y, muy alta } \\
\text { confiabilidad de las mismas }\end{array}$ \\
\hline $\begin{array}{l}\text { 12.- El personal involucrado en las } \\
\text { actividades relacionadas a la } \\
\text { gestión del mantenimiento } \\
\text { preventivo cumple con las } \\
\text { funciones establecidas en el } \\
\text { manual organizacional }\end{array}$ & 2,93 & $\begin{array}{l}\text { Moderada } \\
\text { aplicación }\end{array}$ & 0,26 & $\begin{array}{l}\text { Muy baja dispersión de las } \\
\text { respuestas y, muy alta } \\
\text { confiabilidad de las mismas }\end{array}$ \\
\hline $\begin{array}{l}\text { Promedios de la media y desviación } \\
\text { estándar }\end{array}$ & 2,59 & $\begin{array}{l}\text { Baja } \\
\text { aplicación }\end{array}$ & 0,27 & $\begin{array}{l}\text { Muy baja dispersión de las } \\
\text { respuestas y, muy alta } \\
\text { confiabilidad de las mismas }\end{array}$ \\
\hline
\end{tabular}

Fuente: Elaboración Propia (2019)

Atendiendo a los valores individuales de cada ítem, se tiene baja aplicación al referir los encuestados que la gerencia cuenta con un manual organizacional donde se encuentren establecidas las funciones que cada persona debe desempeñar $(1,90)$; no obstante con moderada aplicación el personal involucrado en las actividades relacionadas a la gestión del mantenimiento preventivo tiene conocimiento acerca de las funciones establecidas en el manual organizacional $(2,93)$ y cumple con las funciones establecidas en el manual organizacional $(2,93)$. Aunado a ello se denota muy baja dispersión de las respuestas $y$, muy alta confiabilidad de las mismas $(0,30 ; 0,26$; $0,26)$.

Estos resultados no son congruentes con lo expresado por Koontz y Weihrich, (2013) quienes consideran que las funciones son las actividades y tareas en forma rutinaria y que al realizarlas, permitirán el cumplimiento de las acciones tácticas que acercan al logro de los objetivos estratégicos. El nivel de coordinación de estas funciones opera entre los niveles operativos y sirve de intermediación y coordinación entre ambos.

Al igual que difieren estos resultados con lo expuesto por el investigador, al considerar que las organizaciones funcionan adecuadamente, al ser organizadas, por esta razón es necesario señalar la importancia de ésta función, que relaciona las actividades necesarias y dispone quien debe desempeñarlas, establece la mejor forma de alcanzar los objetivos propuestos recordando que los objetivos planteados van relacionados según la función que desempeñe el grupo o departamento, para el alcance de estos objetivos combina las tareas en forma lógica y eficiente.

De acuerdo a lo anteriormente descrito, es importante contar con un manual organizacional donde se encuentren claramente definidas las funciones y responsabilidades que cada persona debe desempeñar en las 
actividades del mantenimiento preventivo, para lograr alcanzar la eficacia y eficiencia en la ejecución de dichas actividades y de esta manera cumplir con la misión, visión objetivos dentro del departamento de flota de las gerencias de bienes y servicios de CORPOELEC Región Occidental.

Finalmente, como se puede apreciar en la tabla 7, se presenta el resumen para la dimensión organización del mantenimiento, el cual muestra un valor de 3,56, reflejando de acuerdo a la opinión de los encuestados que existe una alta aplicación con muy baja dispersión de las respuestas y, muy alta confiabilidad de las mismas $(0,41)$, de manera que, se puede afirmar que en el departamento de flota de las gerencias de bienes y servicios de CORPOELEC Región Occidental, la organización de la gestión del mantenimiento preventivo es aplicada a través de la descentralización, organigrama, autoridad y funciones.

Tabla 7. Dimensión Organización del mantenimiento

\begin{tabular}{|c|c|c|c|c|}
\hline Ítems & $\overline{\boldsymbol{X}}$ & Categoría & $\sigma$ & Categoría \\
\hline Descentralización & 4,61 & $\begin{array}{l}\text { Muy alta } \\
\text { aplicación }\end{array}$ & 0,48 & $\begin{array}{l}\text { Muy baja dispersión de las respuestas } \\
\text { y, muy alta confiabilidad de las mismas }\end{array}$ \\
\hline Organigrama & 3,02 & $\begin{array}{l}\text { Moderada } \\
\text { aplicación }\end{array}$ & 0,46 & $\begin{array}{l}\text { Muy baja dispersión de las respuestas } \\
\text { y, muy alta confiabilidad de las mismas }\end{array}$ \\
\hline Autoridad & 4,00 & Alta aplicación & 0,42 & $\begin{array}{l}\text { Muy baja dispersión de las respuestas } \\
\text { y, muy alta confiabilidad de las mismas }\end{array}$ \\
\hline Funciones & 2,59 & Baja aplicación & 0,27 & $\begin{array}{l}\text { Muy baja dispersión de las respuestas } \\
\text { y, muy alta confiabilidad de las mismas }\end{array}$ \\
\hline $\begin{array}{l}\text { Promedios de la media y } \\
\text { desviación estándar }\end{array}$ & 3,56 & Alta aplicación & 0,41 & $\begin{array}{l}\text { Muy baja dispersión de las respuestas } \\
\text { y, muy alta confiabilidad de las mismas }\end{array}$ \\
\hline
\end{tabular}

Fuente: Elaboración Propia (2019)

Sin embargo es importante acotar, que se cumple en mayor jerarquía la descentralización y autoridad, quedando el organigrama y las funciones como elementos que deben ser revisados para lograr un sistema de mantenimiento eficiente y eficaz que permita una combinación voluntaria de personas y recursos diseñados para satisfacer situaciones específicas técnicas, geográficas y de personal, dirigida a la conservación de objetivos y fines del mantenimiento preventivo.

Estos resultados de alta aplicación permiten validar lo planteado por Duffuaa, y otros (2007), quienes señalan que la organización de un sistema de mantenimiento es aquella que incluyen el diseño del trabajo abarcando el contenido de cada tarea y determinando el método que se va a utilizar, las herramientas especiales necesarias y los trabajadores calificados requeridos para el cumplimiento de sus funciones.

De igual manera, se valida la postura del investigador al señalar que la organización de mantenimientos agrupa todas las funciones y actividades en unidades específicas, con base en su similitud. Además, debe existir una coordinación, sincronizando los recursos y los esfuerzos de un grupo social, con el fin de lograr oportunidad, unidad, armonía y rapidez, en desarrollo de los objetivos de la organización.

De acuerdo a lo anteriormente descrito, es importante contar con un manual organizacional donde se encuentren claramente definidas las funciones y responsabilidades que cada persona debe desempeñar en las actividades del mantenimiento preventivo, para lograr alcanzar la eficacia y eficiencia en la ejecución de dichas 
actividades y de esta manera cumplir con la misión, visión y objetivos en el departamento de flota de las gerencias de bienes y servicios de CORPOELEC Región Occidental.

\section{CONCLUSIONES}

En lo que concierne al objetivo, se describió la organización del mantenimiento preventivo aplicado en las gerencias de bienes y servicios de CORPOELEC Región Occidental, observándose que el personal involucrado en las actividades señala que dentro del proceso, los indicadores descentralización, autoridad, y organigrama se aplican dentro del objetivo. Sin embargo se requiere mejorar el resultado obtenido en el promedio del indicador funciones puesto que éste tiene baja aplicación.

Debe mencionarse que dada esta categoría de alta aplicación, el investigador concluye que los departamentos analizados poseen alto criterio de aplicación de la organización del mantenimiento y que el mismo está estructurado bien sea por departamentos, área $\circ$ de manera centralizada, tomando en cuenta los recursos de que dispone las gerencias de bienes y servicios de forma que pueda funcionar acorde a lo previsto en la planificación.

En tal sentido, se observó específicamente, que en la organización del mantenimiento preventivo se conocen las decisiones y actividades que se llevan a cabo, por lo tanto quienes gerencian el mantenimiento poseen la propiedad de mandar y a la obligación de que sus decisiones tomadas sean cumplidas por otros, pero dentro de una estructura de mantenimiento que no siempre se tiene una idea de cómo está estructurada, traduciéndose esto en el poco cumplimiento de las tareas necesarias para cumplir con objetivos prefijados según el manual organizacional. En síntesis, se concluye que el mantenimiento debe organizarse para dar respuesta rápida, efectiva y cónsona con la realidad organizativa.

\section{REFERENCIAS BIBLIOGRAFICAS}

Creus, A. (2006). Fiabilidad y Seguridad. Su aplicación en procesos industriales. Segunda Edición. Editorial Marcombo, S.A. España

Decreto-Ley Nro. 5.330 (2007). Decreto con rango, valor y fuerza de ley orgánica de reorganización del sector eléctrico. Disponible en: http://www.cpzulia.org/ARCHIVOS/Decr 5 330 Corp Elect Nac 3107 07.pdf

Duffuaa, S; Raouf, A. y Dixon; J. (2007). Sistemas de mantenimiento: planeación y control. Editorial Limusa Wiley. México.

Garrido, S. (2003). Organización y gestión Integral de Mantenimiento. Editorial Díaz de Santos, s.a., España

González, R. (2011). Mantenimiento Industrial (organización, gestión y control). Edit Librería Alsina. Buenos Aires.

Koontz, H. y Weihrich, H. (2013). Elementos de administración: un enfoque internacional y de innovación. McGraw Hill Education México

Milano, T (2010). Planificación y Gestión del Mantenimiento Industrial. Un Enfoque Estratégico y Operativo. 1ํㅡ Edición, Editorial Panapo. Venezuela

Nava, J. (2008). Teoría de mantenimiento: definiciones y organización. Mérida, Venezuela: Universidad de los Andes

Norma venezolana (1993). COVENÍN 3049. 93. Mantenimiento. Definición. Comisión Venezolana de Normas Industriales, Caracas, Venezuela.

Robbins, S. y Decenzo, D. (2002). Fundamentos de Administración. Tercera Edición. Editorial Pretince Hall. México 